Research Article

\title{
Current concept of management of supracondylar femur fracture: retrograde femoral nail or distal femoral locking plate
}

\author{
Chaitanya Krishna*, Raja Vinay Shankar
}

Department of Orthopedics, Medial College and Hospital, West Bengal University of Health Sciences, Kolkata, India

Received: 17 February 2016

Revised: 12 April 2016

Accepted: 03 June 2016

*Correspondence:

Dr. Chaitanya Krishna,

E-mail: chatconfident@gmail.com

Copyright: (c) the author(s), publisher and licensee Medip Academy. This is an open-access article distributed under the terms of the Creative Commons Attribution Non-Commercial License, which permits unrestricted non-commercial use, distribution, and reproduction in any medium, provided the original work is properly cited.

\begin{abstract}
Background: This study provides an update on the management of supracondylar femur fractures by distal femoral locking plate and retrograde femoral nail.

Methods: 20 patients in each group with median age of 47 years treated with plate fixations and nailing for supracondylar femur fractures was followed up for a period of 12 months.

Results: Distal femoral nail has shorter union time than distal femoral locking plate, group of patients with distal

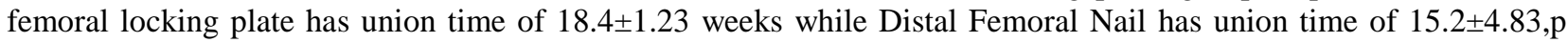
value was 0.007 which is significant. Total range of motion and extensor lag is same. The total amount of knee flexion in distal femoral Locking Plate is 104.75 \pm 8.19 , while in Distal Femoral Nail is 109.5 \pm 11.46 , p value is 0.14, which is not significant .Functional results are same.

Conclusions: It can be concluded that results are better with both the technique, provided done surgically correct. The plate can be adapted to all fractures, while retrograde nailing is better adapted to extra-articular fractures.
\end{abstract}

Keywords: Distal femoral nail, Distal femoral locking plate, Supracondylar femur fracture, Femur

\section{INTRODUCTION}

In the early 1960 s, there was a great reluctance towards operative management of this fracture because of high incidence of infection, non-union, malunion, inadequate fixation and lack of proper instruments, implant as well as antibiotics. Then, the traditional management of displaced fracture supracondylar of femur was along the principle of Watson Jones and John Charnley. ${ }^{1,2}$ This comprised of skeletal traction, manipulation of fracture and external immobilization in the form of casts and cast bracings. These methods however, met with problems like deformity, shortening, prolonged bed rest, knee stiffness, angulation, joint incongruity, malunion, quadriceps wasting, knee instability and post-traumatic osteoarthritis.
The trend of open reduction and internal fixation has become evident in the recent years, with good results being obtained with the AO blade plate, dynamic condylar screw and other implant systems like intramedullary supracondylar nails and recently locking compression plate. During application of AO blade plate or dynamic condylar screw, creates rotational movement at the fracture site that causes pulling off the blade plate or condylar screws leading to fatigue fracture of the plates. Also, the presence of osteoporotic bone leads to fixation failures with screws and plates cutting of the soft bone.

During application of distal femoral plate, the shaft of femur is often pulled laterally displacing the line of weight bearing, lateral to the anatomical axis of condyle. The obvious advantage of an intramedullary device is that 
it aligns the femoral shaft with condyles reducing the tendency to place varus movement at the fracture site but at the same time entry from the intra-articular site causes knee stiffness. Locking compression plate has the advantage of combination of conventional compression plating and locked plating techniques which enhances the plate osteosynthesis.

The advantage of an intramedullary device is that it maintains the anatomical axis, and because the bending movement of an intramedullary device is substantially reduced, failure of fixation in osteoporotic bone should be less. In addition, a retrograde intramedullary supracondylar nail has got distinct advantages of preservation of fracture hematoma, decreased blood loss, minimal soft tissue dissection, less operative time and reduced rate of infection. Locking compression plate has anatomically pre-contoured built reduces soft tissue problems and acts as internal external fixator. In addition, a locking compression plate has got distinct advantages of uni-cortical fixation and least chance of plate back out as the screw gets locked to the plate. Further, minimal soft tissue injury occurs when closed reduction is done and MIPO technique is used. The purpose of this study is to evaluate the results of supracondylar fracture of femur, treated by close/open reduction and internal fixation using retrograde intramedullary nail and locking plate and analysis of their results.

\section{METHODS}

A prospective, double blinded, longitudinal, single cohort study with simple random sampling of patients treated with plate fixations or distal femoral nailing for supracondylar femur fractures. 20 case each for plate fixation and distal femoral nailing. There were 40 cases in this study. The study was conducted at department of orthopedics, medical college and hospital, Kolkata, India. We studied our patients who fitted our criteria for the study. A written consent was obtained from all the patients.The study was done after obtaining clearance from our institutional ethical committee before the subjects are recruited and recommended.

\section{Parameters to be studied are}

- Assessment by using standard radiographic criteria for time of union, incidence of infection and knee function with a functional Knee Score

- Incidence of complication after surgery viz. Implant failure, wound infection, delayed union

- Quality of life and pain status at follow up.

Inclusion criteria are skeletally mature patients, fracture of supracondylar femur without intra articular extension, fracture requiring operative treatment amenable to either distal femoral nailing or locking plate and informed consent obtained. Exclusion criteria are fracture of the supracondylar femur with intra-articular extension, open fracture involving supracondylar femur, pathological fracture, known metabolic bone disease, existing deformity in the affected limb that would complicate plating or distal femoral nailing and immunocompromised patient. Pre-operative preparation Fractures were classified with the help of radiographs according to the AO-ASIF classification. Preoperative calculation was done on radiographs to ascertain the length of supracondylar nail, maximum possible diameter and lengths of interlocking bolts after subtraction of the magnification factor. After induction of patient with regional or general anaesthesia, a bolster was kept underneath the knee so that knee is in 500 to 600 flexion. Cotton padding was always placed to prevent neurovascular insult.

\section{Operative procedure}

\section{For intra-medullary nailing}

A midline incision of $4 \mathrm{~cm}$ was taken from inferior pole of patella up to tibial tuberosity. A straight bone awl was inserted into the joint through the split tendon and positioned against the inter-condylar notch. The femoral attachment of Posterior Cruciate Ligament is palpated and the bone awl is kept just anterior to the Posterior cruciate ligament attachment. The bone awl was then removed and guide wire passed through the entry point. The fracture was reduced under image intensifier control and guide wire passed in proximal fragment. The nail was then inserted over the guide wire through the entry point made previously through distal and then proximal fragment. Either single or both holes were locked proximally. Similarly, the distal holes were locked in one, two or three numbers.

The important step in the evolution of plating distal femur fractures was the introduction of locking plates. Distal femoral locking plates are anatomically contoured and have multiple locking screw options distally to allow for secure fixation in the typical short condylar segment. When using the plate as a reduction aid, the compression screw draws the bone towards the plate and uses the contour of the plate to reduce the fracture in the coronal plane. Reduction of the fracture was assisted keeping folded pillow below the knee which prevented posterior angulation of distal fragment with manual traction. Once the fracture is reduced, supplemental locking screws were then added to create a fixed-angle construct.

\section{RESULTS}

In our study, 40 supracondylar femoral fractures were treated. All cases were fresh, 35 patients were males and 5 patients were females. The median age was 47 years ranging from 28-70 years. 35 of the fractures were caused by road traffic accidents 3 were due to fall and 2 were due to assault. 28 patients were with fracture on right side and 12 on left side. 
Average knee flexion in this study was 104 degree with more than $50 \%$ patients having knee range of motion more than $110^{\circ}$. Out of 40 patients, 3 patients had shortening, 2 patients with shortening of $15 \mathrm{~mm}$ and 1 patient with shortening of $10 \mathrm{~mm}$. In this study, very few patients had significant varus/ valgus mal-alignment with 2 patients had deep infection which was treated with debridement and antibiotics. The duration of follow-up was 12 months.

Group of patients with distal femoral locking plate has union time of $18.4 \pm 1.23$ weeks while Distal Femoral Nail has union time of $15.2 \pm 4.83$. p value was 0.007 which is significant. This value shows that distal femoral nail has shorter union time. The total amount of knee flexion in distal femoral locking plate is $104.75 \pm 8.19$, while in distal femoral nail is $109.5 \pm 11.46$. $p$ value is 0.14 , which is not significant. So both the technique has equal amount of knee flexion. The total amount of extensor lag in both the groups is also same as distal femoral locking plate has extensor lag of $5.7 \pm 4.31$ while distal femoral nail has extensor lag of $5 \pm 6.28$. $p$ value is $<0.05$ which is not significant.

Neer rating (0-100 points) is done for both the groups, with distal femoral locking plate having $79.15 \pm 12.05$ while distal femoral nail have $76.45 \pm 11.27 .^{3}$ Results are found to be not significant. Functional outcome was not significant in both the groups.

Table 1: Functional outcome in two groups of distal femoral locking plate and distal femoral nail.

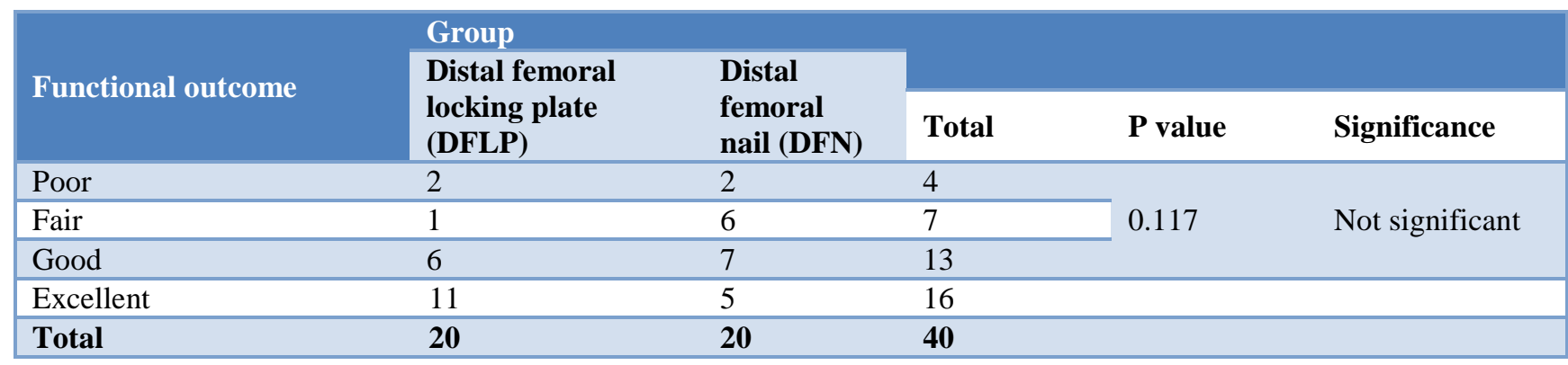

\section{DISCUSSION}

Fractures of the distal femur often pose challenges to the trauma surgeon because of the proximity to the knee joint. In the younger age group, the injury is usually a result of high velocity vehicular crashes. In older patients with osteoporotic bone, it is often due to low energy injuries especially falls. In either case, the fracture is usually comminuted. Treatment requires skill and meticulous technique for good outcome. A variety of devices have been used in the treatment of supracondylar femoral fractures. The locking plate relies on the principles of open reduction, absolute stability and interfragmentary compression to achieve union. The technique of retrograde nailing uses indirect reduction of the metaphyseal fracture component, offering relative stability and a less invasive approach.

Internal fixation of supracondylar femoral fractures using distal femoral locking plate produces good results in most cases. However, the incidence of bone infections is high.

In case of distal femoral nail, there is a trend for patients undergoing retrograde nailing to complain of more pain and to require revision surgery for removal of implants. There seems to be a tendency towards progression to arthritis particularly in fractures treated by distal femoral nail. The quality of the surgical technique is the primary factor, and the only guarantee of obtaining good radiological and clinical results in supracondylar femoral fractures. Mini-invasive treatment (nailing or plates) seems to provide better results.

Markmiller et al prospectively compared the outcome of condylar blade plate and retrograde intramedullary nail. ${ }^{4}$ At 12 months; no statistically significant differences were noted with non-union, fixation failure, infection and secondary surgical procedure.

Hierholzer et al confirmed these results in a retrospective series of 115 fractures comparing retrograde nailing $(\mathrm{n}=$ 59) and mini-invasive locking plate $(n=56) .^{5}$ The authors describe the indications for each technique: the plate can be adapted to all fractures, while retrograde nailing is better adapted to extra-articular fractures. They emphasize that high quality results are more dependent upon the surgical technique than the choice of implant.

Hartin et al did not observe any difference in functional recovery in a randomized comparison of the treatment of extra-articular fractures by retrograde intramedullary nailing and blade plate. ${ }^{6}$ The only element observed was more frequent pain in the knee in the retrograde nailing group, so that fixation material had to be removed in $25 \%$ of the cases. 
For Thompson et al, statistical results for the rate of surgical revision and the rate of malunion are better for retrograde intramedullary nailing. The rates of infection and nonunion were higher in the open internal fixation group. Zlowodzki et al compared LCP, blade plates, and retrograde nailing in extra-articular fractures. ${ }^{7}$ Strength under axial compression was better with the LCP system than with the blade plate or nailing, by 34 and $13 \%$ respectively, but strength under torsion was reduced. ${ }^{8-12}$ The authors observed better distal fixation with the LCP system with loss of distal fixation in only one LCP plate (6\%), three blade plates $(38 \%)$ and eight losses with retrograde intramedullary nailing $(100 \%) .{ }^{13,14}$

\section{CONCLUSION}

The goal of this study was to provide an update on the management of these fractures by two modalities of treatment. The technical details and the indications of both the surgical treatments are described and from our study it can thus be concluded that results are better with both the techniques. The surgical technique must be rigorous and the biomechanical qualities of both the implants must be understood to prevent the development of major complications. The plate can be adapted to all fractures, while retrograde nailing is better adapted to extra-articular fractures. They emphasize that high quality results are more dependent upon the surgical technique than the choice of implant. On the other hand, results comparing retrograde nailing and classic open internal fixation are clear.

Funding: No funding sources

Conflict of interest: None declared

Ethical approval: The study was approved by the institutional ethics committee

\section{REFERENCES}

1. Wilson JN. Watson Jone's: Fractures and joint injuries. $6^{\text {th }}$ ed; 1982:1003-070.

2. John C. The closed treatment of common fractures. $3^{\text {rd }}$ ed; 2003:197-204.

3. Neer CS, Gratham SA, Shelton ML. Supracondylar fractures of adult femur. JBJS. 1967;49(A):591-613.
4. Markmiller M, Konrad G, Südkamp N. Femur LISS and distal femoral nail for fixation of distal femoral fractures: are there differences in outcome and complications? Clin Orthop Relat Res. 2004;426:252-7.

5. Hierholzer C, von Rüden C, Pötzel T, Woltmann A, Bühren V. Outcome analysis of retrograde nailing and less invasive stabilization system in distal femoral fracture: a retrospective analysis. Indian $\mathbf{J}$ Orthop. 2011;45:243-50.

6. Hartin NL, Harris I, Kaushik H. Retrograde nailing versus fixed angled blade plating for supra-condylar femoral fractures: a randomized controlled trial. ANZ J Surg. 2006;76:290-4.

7. Zlowodzki M, Williamson RS, Cole PA. Biomechanical evaluation of the less invasive stabilization system, angled blade plate, and retrograde intramedullary nail for the internal fixation of distal femur fractures. J Orthop Trauma. 2004;18:494-502.

8. Crist BD, Della Rocca GJ, Murtha YM. Treatment of acute distal femur fractures. ORTHOPEDICS. 2008;31:681.

9. Acharya KN, Rao MR. Retrograde nailing for distal third femoral shaft fractures: a prospective study. J Orthop Surg (Hong Kong). 2006;14:253-8.

10. Kanabar P, Kumar V. Less invasive stabilisation system plating for distal femoral fractures. J Orthop ( Hong Kong) . 2007;15:299-302.

11. Nayak RM, Koichade MR. Minimally invasive plate osteosynthesis using a locking compression plate for distal femoral fractures. J Orthop (Hong Kong). 2011;19:185-90.

12. Higgins TF, Pittman G. Biomechanical analysis of distal femur fracture fixation: fixed angle screw plate construct versus condylar blade plate. J Orthop Trauma. 2007:2143-6.

13. Lujan TJ, Henderson CE. Locked plating of distal femur fractures leads to inconsistent and asymmetric callus formation. J Orthop Trauma. 2010;24:156-62.

14. Ehlinger M, Adam P. Minimally invasive fixation of distal extra-articular femur fractures with locking plates: limitations and failures. Orthop Traumatol Surg Res. 2011;97:668-74.

Cite this article as: Krishna C, Shankar RV. Current concept of management of supracondylar femur fracture: retrograde femoral nail or distal femoral locking plate. Int Surg J 2016;3:1356-59. 